\title{
On the Statistical Meaning of Complex Numbers in Quantum Mechanics
}

\author{
L. Accardi \\ Istituto di Matematica dell'Università - Cagliari, Italia \\ Istituto di Cibernetica del C.N.R. - Arco Felice, Napoli, Italia \\ A. Fedullo \\ Istituto di Scienze dell'Informazione dell'Università - Salerno, Italia
}

(ricevuto il 2 novembre 1981)

Summary. - Bell's inequality is a necessary condition for the existence of a classical probabilistic model for a given set of correlation functions. This condition is not satisfied by the quantum-mechanical correlations of two-spin systems in a singlet state.

We give necessary and sufficient conditions, on the transition probabilities, for the existence of a classical probabilistic model. We also give necessary and sufficient conditions for the existence of a complex (respectively real) Hilbert space model.

Our results apply to individual-spin systems hence they need no "locality" assumption. When applied to the quantum-mechanical transition probabilities, they prove not only the necessity of a nonclassical probabilistic model, but also the necessity of using complex rather than real Hilbert spaces.

Statement of the problem. - The problem of understanding the empirical basis of the quantum-mechanical formalism has been studied by many authors, both physicists and mathematicians (cf. the excellent survey ${ }^{(1)}$ and the bibliography therein). Recently a new approach to this problem has been proposed (cf. ${ }^{(2-4)}$ ) in which one considers the conditional (i.e. transition) probabilities as the basic empirical data from which the mathematical model should be deduced. The main idea of this approach is to classify the probabilistic models, both Kolmogorovian and non-Kolmogorovian, according to statistical invariants, which are expressed in terms of the transition probabilities.

(1) A.S. Wightman: Hilbert's sixth problem: mathematical treatment of the axioms of physics, in Mathematical Developments Arising from Hilbert Problems. Proceedings Symposia in Pure Mathematics, vol. 28 (Providence, 1976).

(2) L. Accardi: Topics in quantum probability, to appear Rep. Phys.

(3) L. Accardi: Non-Kolmogorovian probabilities, in Rendiconti del Seminario Matematico dell'Università e del Politecnico di Torino.

(4) L. Accardi: Foundations of quantum probability, invited address to the III Vilnius Conference, Probability and Mathematical Statistics, Vilnius, June 1981 (to appear). 
In the present work the statistical invariants for some simple systems are explicitly computed and it is shown that they allow us to distinguish among Kolmogorovian, realHilbert-space and complex-Hilbert-space models. These statistical invariants depend only on the transition probabilities which can be considered as empirical data, and it is very simple to produce examples of quantum systems such that the statistical invariants associated to their transition probabilities do not allow any Kolmogorovian or real-Hilbert-space model, but obviously allow a complex-Hilbert-space model.

Therefore the method of statistical invariants allows us to solve the problem of "... singling out in full generality the empirical basis for the choice of complex numbers in quantum theory..." (cf. $\left.{ }^{(5)}, \S 8-5\right)$.

The problem of the meaning of the number field in quantum theory has been studied, from different points of view, by several authors (cf. ${ }^{(6-9)}$ ).

Let us now state the problem in a precise mathematical form. Let $A, B, C, \ldots$ denote some (a finite or an infinite set) observable quantities with values $\left(a_{\alpha}\right),\left(b_{\beta}\right),\left(c_{\gamma}\right)$, respectively. We will assume that $\alpha, \beta, \gamma, \ldots=1,2, \ldots, n$ for some $n \leq+\infty$, independent of $A, B, C, \ldots$ and that $a_{\alpha}, b_{\beta}, c_{\gamma}, \ldots \in \mathbf{R}$. Consider the transition probabilities

$$
P\left(A=a_{\alpha} \mid B=b_{\beta}\right), \quad P\left(B=b_{\beta} \mid C=c_{\gamma}\right), \quad P\left(C=c_{\gamma} \mid A=a_{\alpha}\right), \ldots,
$$

where $P\left(A=a_{\alpha} \mid B=b_{\beta}\right)$ denotes the probability that $A$ takes the value $a_{\alpha}$ conditioned by the fact that $B$ is known to assume the value $b_{\beta}$. Since we are interested in the comparison between the classical and the quantum-mechanical situation, we will assume that the transition probabilities satisfy the symmetry conditions

$$
P\left(A=a_{\alpha} \mid B=b_{\beta}\right)=P\left(B=b_{\beta} \mid A=a_{\alpha}\right), \ldots
$$

(where, here and in the following, the dots after a relation will stand for the same relation written for all remaining obsevables). It will also be assumed, although this is not essential for our goals, that for each $\alpha, \beta, \ldots$

$$
P\left(A=a_{\alpha} \mid B=b_{\beta}\right)>0, \ldots .
$$

We will say that the transition probabilities (1) admit a Komogorovian model, if there exist

1) a probability space $(\Omega, \mathcal{O}, \mu)$,

2 ) for each observable $A, B, C, \ldots$, a measurable partition of $\Omega-\left(A_{\alpha}\right),\left(B_{\beta}\right),\left(C_{\gamma}\right), \ldots$ such that, for each $\alpha, \beta, \gamma, \ldots$

$$
P\left(A=a_{\alpha} \mid B=b_{\beta}\right)=\frac{\mu\left(A_{\alpha} \cap B_{\beta}\right)}{\mu\left(B_{\beta}\right)} ; \ldots .
$$

(5) J.M. Jauch: Foundations of Quantum Mechanics (Reading, Mass., 1968).

(6) G. Emch: Helv. Phys. Acta, 36, 739, 770 (1963).

(7) D. Finkelstein, J.M. Jauch, S. Shiminovich and D. Speiser: J. Math. Phys. (N.Y.), 3, 207 (1962).

(8) D. Filkestein, J.M. Jauch, S. Schiminovich and S. Speiser: J. Math. Phys. (N.Y.), 4, 788 (1963).

(9) E.C.G. Stueckelberg: Helv. Phys. Acta, 33, 727 (1960); E.C.G. Stueckelberg and M. Guenin: Helv. Phys. Acta, 34, 621 (1961); E.C.G. Stueckelberg, M. Guenin, C. Piron and H. Ruegg: Helv. Phys. Acta, 34, 675 (1961); E.C.G. Stueckelberg and M. Guenin: Helv. Phys-Acta, 35 (1962). 
We will say that the transition probabilities (1) admit a complex (respectively, real) Hilbert space model, if there exist

1) a complex (respectively real) Hilbert space $\mathcal{H}$ of dimension $n$;

2) for each observable $A, B, C, \ldots$, an orthonormal basis of $\mathcal{H}-\left(\varphi_{\alpha}\right),\left(\psi_{\beta}\right),\left(\chi_{\gamma}\right), \ldots$ : such that, for each $\alpha, \beta, \gamma, \ldots$

$$
P\left(A=a_{\alpha} \mid B=b_{\beta}\right)=\left|\left\langle\varphi_{\alpha}, \psi_{\beta}\right\rangle\right|^{2}, \ldots
$$

In this case we say that the given orthonormal bases realize the corresponding transition probabilities.

Thus, for a given set of transition probabilities $P\left(A=a_{\alpha} \mid B=b_{\beta}\right), \ldots$, which we can consider as "experimental data", it is quite natural to ask the following questions:

Problem 1. Do they always admit a Kolmogorovian model?

Problem 2. Do they admit a Hilbert space model but not a Kolmogorovian one, or conversely?

Problem 3. Do they admit both a Kolmogorovian and a Hilbert space model?

Problem 4. Do they admit a complex Hilbert space, but neither a real Hilbert space nor a Kolmogorovian model?

An example of transition probabilities admitting a complex Hilbert space, but not a Komogorovian model is implicit in Wigner's proof of Bell's inequality ${ }^{(10)}$ (in Bell's original proof, correlations rather than conditional probabilities are considered $\left.{ }^{(11)}\right)$. In general one can prove $\left(\right.$ cf. $\left.^{(2,4)}\right)$ that if the conditional probabilities satisfy (2) - as is always the case in quantum theory - there is always a Kolmogorovian model for two observables, but generically not for three or more (for this reason, henceforth, we will always consider three observables $A, B, C)$. In the following it will be shown that the method of statistical invariants, mentioned above, allows us to give a complete solution of Problems 1-4-and of similar questions - in the case of three observables $A, B, C$ each of which assumes two real values (cf. ${ }^{(12)}$ for the extension to the case of an arbitrary finite number of values).

Kolmogorovian models. - Let $A, B, C$ be three $n$-valued observables.

Propositin 1 (cf. $\left.{ }^{(3)}\right)$. In the notations and assumptions introduced above the three transition matrices with coefficients

$$
P\left(A=a_{\alpha} \mid B=b_{\beta}\right), \quad P\left(B=b_{\beta} \mid C=c_{\gamma}\right), \quad P\left(C=c_{\gamma} \mid A=a_{\alpha}\right)
$$

$(\alpha, \beta, \gamma=1, \ldots, n)$ admit a Kolmogorovian model if and only if there are $n^{3}$ real numbers $\left(\Gamma_{\alpha, \beta, \gamma}\right)$ such that

$$
\Gamma_{\alpha, \beta, \gamma} \geq 0
$$

(10) E.P. Wigner: Am. J. Phys., 38, 1005 (1970).

(11) J.S. Bell: Physics, 1, 195 (1964).

(12) L. Accardi and A. Fedullo: Statistical invariants for the probabilistic models of finite-valued observables (in preparation). 


$$
\sum_{\gamma} \Gamma_{\alpha, \beta, \gamma}=P\left(A=a_{\alpha} \mid B=b_{\beta}\right)
$$

$$
\sum_{\beta} \Gamma_{\alpha, \beta, \gamma}=P\left(A=a_{\alpha} \mid C=c_{\gamma}\right)
$$

$$
\sum_{\alpha} \Gamma_{\alpha, \beta, \gamma}=P\left(B=b_{\beta} \mid C={ }_{\gamma}\right) .
$$

Proof. Necessity. Let $(\Omega, \mathcal{O}, \mu),\left(A_{\alpha}\right),\left(B_{\beta}\right),\left(C_{\gamma}\right)$ be a Kolmogorovian model for the given transition matrices. Then the symmetry condition (2) implies that ( cf. $^{(2)}$ )

$$
\mu\left(A_{\alpha}\right)=\mu\left(B_{\beta}\right)=\mu\left(C_{\gamma}\right)=\frac{1}{n}, \quad \forall \alpha, \beta, \gamma,
$$

hence

$$
\frac{1}{n} P\left(A=a_{\alpha} \mid B=b_{\beta}\right)=\mu\left(A_{\alpha} \cap B_{\beta}\right)=\sum_{\gamma} \mu\left(A_{\alpha} \cap B_{\beta} \cap C_{\gamma}\right),
$$

which implies (6) and (7) with $\Gamma_{\alpha, \beta, \gamma}=n \cdot \mu\left(A_{\alpha} \cap B_{\beta} \cap C_{\gamma}\right)$. (8) and (9) are obtained in a similar way.

Conversely, let $\left(\Gamma_{\alpha, \beta, \gamma}\right)$ be $n^{3}$ numbers which satisfy (6)-(9). Then on the set $\Omega$ of all triples $(\alpha, \beta, \gamma)(\alpha, \beta, \gamma=1, \ldots, n)$ one can define the measure $\mu$ by

$$
\mu(\alpha, \beta, \gamma)=\frac{1}{n} \Gamma_{\alpha, \beta, \gamma}
$$

and the partitions

$$
A_{\alpha}=\bigcup_{\beta \gamma}(\alpha, \beta, \gamma), \quad B_{\beta}=\bigcup_{\alpha \gamma}(\alpha, \beta, \gamma), \quad C_{\gamma}=\bigcup_{\alpha \beta}(\alpha, \beta, \gamma)
$$

and it is easy to check that they provide a Kolmogorovian model for the given transition matrices.

From now on we will limit our discussion to the case in which the three observables $A, B, C$ take only two (arbitrary) values. The associated transition probability matrices will be denoted

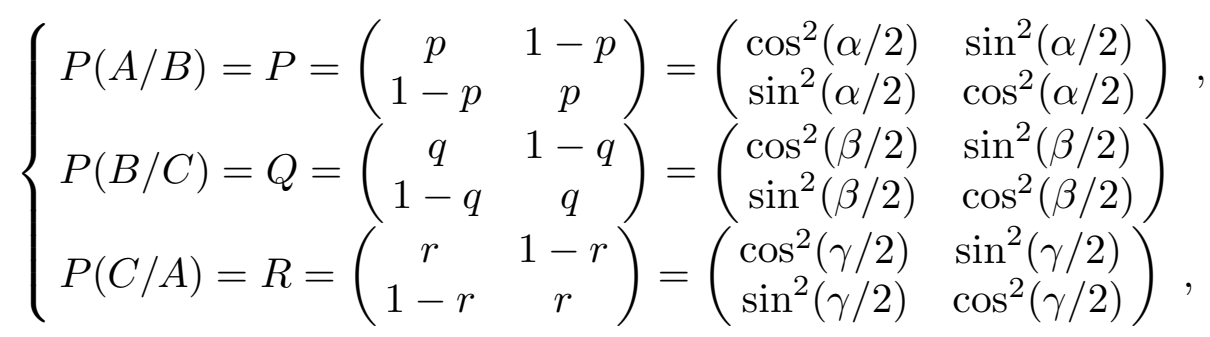


where, because of the assumption (3), $0<p, q, r<1$ and therefore the angles $\alpha, \beta, \gamma$ can always be chosen to satisfy

$$
0<\alpha, \beta, \gamma<\pi
$$

Theorem 2. The transition matrices $P, Q, R$ defined by (10) admit a Kolmogorovian model, if and only if

$$
|p+q-1| \leq r \leq 1-|p-q|
$$

Remark. The asymmetry between the parameters $r$ and $p, q$ in condition (12) is only apparent. The inequalities (12) provide a simple example of a statistical invariant for Kolmogorovian models.

Proof.- We show that (12) is a necessary and sufficient condition for the solvability of (6)-(9) with $n=2$ and $P(A / B), P(B / C), P(C / A)$ given by $(10)$.

In our case (7), (8), (9) yield twelve equations in the eight unknowns $\Gamma_{\alpha, \beta, \gamma}(\alpha, \beta, \gamma=$ $1,2)$. Using this equations, we express seven of these unkwons as functions of $\Gamma_{2,1,1}$ and $p, q, r$ obtaining

$$
\begin{cases}\Gamma_{1,1,1}=q-\Gamma_{2,1,1}, & \Gamma_{1,2,1}=r-q+\Gamma_{2,1,1} \\ \Gamma_{1,1,2}=p-q+\Gamma_{2,1,1}, & \Gamma_{2,1,2}=1-p-\Gamma_{2,1,1} \\ \Gamma_{1,2,2}=1-r-p+q-\Gamma_{2,1,1}, & \Gamma_{2,2,1}=1-r-\Gamma_{2,1,1} \\ \Gamma_{2,2,2}=\Gamma_{2,1,1}-1+p+r . & \end{cases}
$$

The positivity conditions (6) are then equivalent to

$$
\begin{cases}q \geq \Gamma_{2,1,1} \geq 1-p-r, & 1-p \geq \Gamma_{2,1,1} \geq q-r \\ 1-q+p-q \geq \Gamma_{2,1,1} \geq 0, & 1-r \geq \Gamma_{2,1,1} \geq q-p\end{cases}
$$

Thus any number $\Gamma_{2,1,1}$ satisfying (14) defines, through (13) and Proposition 1, a Kolmogorovian model for the given transition matrices. Since, as shown by elementary computations, (12) is the necessary and sufficient condition for the solvability of the system of inequalities (14), the theorem is proved.

Remark. Under our assumption $0<p, q, r<1$, it is easy to verify that if a solution for the system (14) exists, then there will be infinitely many solutions. In other terms, the Kolmogorovian model, when existing, will not be unique up to stochastic equivalence. This is intuitively obvious, since the transition probabilities provide information only on the joint probabilities of pairs of the observables $A, B, C$, and it is well known that the stochastic equivalence class of the process $A, B, C$ is determined by the joint distribution of the triple $A, B, C$. 
Spin models. - Our discussion of problem 4 will consist of three main steps: 1) first we characterize those triples of transition probabilities which admit a complex-Hilbertspace model such that the three orthonormal bases satisfying (5) can be chosen to be eigenvectors of three spin matrices - these will be called "spin models"; 2) we then find a necessary condition for a triple of transition matrices to admit a complex-Hilbert-space realization and show that this condition is equivalent to the one characterizing the existence of a spin model. From this we can easily deduce that all complex Hilbert space models for the given transition matrices are spin models; 3) finally we characterize those triples of transition matrices which admit a real Hilbert-space model and show that there are many triples of transition matrices which admit a complex Hilbert space model, but do not admit neither a real Hilbert space nor, a Kolmogorovian model.

Let $P, Q, R$ be the transition matrices defined by (10), (11). In the following, whenever we speak of a complex Hilbert space model for $P, Q, R$ we shall always suppose that an orthonormal basis has been singled out in $\mathcal{H} \cong \mathbf{C}^{2}$. With respect to this basis we define the Pauli matrices

$$
\sigma_{1}=\left(\begin{array}{ll}
0 & 1 \\
1 & 0
\end{array}\right), \quad \sigma_{2}=\left(\begin{array}{cc}
0 & -i \\
i & 0
\end{array}\right), \quad \sigma_{3}=\left(\begin{array}{cc}
1 & 0 \\
0 & -1
\end{array}\right)
$$

and the spin operators

$$
\sigma \cdot a=\sigma_{1} a_{1}+\sigma_{2} a_{2}+\sigma_{3} a_{3}
$$

where

$$
a=\left(a_{1}, a_{2}, a_{3}\right) \in S^{(2)}=\left\{\left(a_{1}, a_{2}, a_{3}\right) \in \mathbf{R}^{3}: \sum_{1}^{2} a_{j}^{2}=1\right\} .
$$

We will always refer to this fixed "canonical" basis when speaking of "components" of a vector in $\mathcal{H}$ without further specification. The eigenvector of $\sigma \cdot a$ corresponding to the eigenvalue +1 (respectively, -1 ) will be denoted $\psi_{1}(a)$ (respectively, $\psi_{2}(a)$ ). A complex Hilbert space model for $P, Q, R$ will be called a spin model if there exist three vectors $a, b, c \in S^{(2)}$ such that the three orthonormal basis $\left(\psi_{\alpha}(a)\right),\left(\psi_{\beta}(b)\right),\left(\psi_{\gamma}(c)\right)$ realize the matrices $P, Q, R$ in the sense of the equalities (5). Since for each $\alpha, \beta, \gamma$

$$
\begin{aligned}
& \left|\left\langle\psi_{1}(a), \psi_{1}(b)\right\rangle\right|^{2}=\cos ^{2}(\widehat{a b} / 2), \ldots \\
& \left|\left\langle\psi_{1}(a), \psi_{2}(b)\right\rangle\right|^{2}=\sin ^{2}(\widehat{a b} / 2), \ldots
\end{aligned}
$$

(where $\widehat{a b}$ denotes the angle between $a$ and $b$ ), one easily verifies that a spin model for $P$, $Q, R$ exists if and only if there exist three vectors $a, b, c \in S^{(2)}$, such that

$$
\cos \alpha=\cos \widehat{a b}, \quad \cos \beta=\cos \widehat{b c}, \quad \cos \gamma=\cos \widehat{c a} .
$$

Proposition 3. Three vectors $a, b, c \in S^{(2)}$ satisfying (18) exist if and only if

$$
\cos ^{2} \alpha+\cos ^{2} \beta+\cos ^{2} \gamma-1 \leq 2 \cos \alpha \cos \beta \cos \gamma
$$


Proof .- Two vectors $a, b \in S^{(2)}$ satisfying the first of the equalities (18) always exist, and we can choose co-ordinates in $\mathbf{R}^{3}$ so that $a=(1,0,0), b=(\cos \alpha, \sin \alpha, 0)$.

The existence of a $c \in S^{(2)}$ satisfying the first of the equalities (18) is therefore equivalent to the existence of a vector $\left(c_{1}, c_{2}, c_{3}\right) \in \mathbf{R}^{3}$, satisfying

$$
c_{1}=\cos \gamma, \quad c_{1} \cos \alpha+c_{2} \sin \alpha=\cos \beta, \quad \sum_{1}^{3} c_{j}^{2}=1
$$

and (19) is the necessary and sufficient condition for this occurrence.

Proposition 4. i) If a triple of coplanar vectors satisfies (18), then all the triples with this property are made of coplanar vectors. ii) If there is a triple of vectors $a, b, c \in S^{(2)}$ which are not coplanar satisfy (18), then for aby other triple $a^{\prime}, b^{\prime}, c^{\prime} \in S^{(2)}$ satisfying (18) one has

$$
\widehat{a b}=\widehat{a^{\prime} b^{\prime}}, \quad \widehat{b c}=\widehat{b^{\prime} c^{\prime}}, \quad \widehat{c a}=\widehat{c^{\prime} a^{\prime}} .
$$

Proof .- Any three vectors $a, b, c \in S^{(2)}$ are such that

$$
\begin{gathered}
|\widehat{a b}-\widehat{b c}| \leq \widehat{c a} \leq \widehat{a b}+\widehat{b c} \\
\widehat{a b}+\widehat{b c}+\widehat{c a} \leq 2 \pi
\end{gathered}
$$

(cf. $\left.^{(13)}, \S \mathrm{X}-689,690\right)$ and the equality in (23) holds if and only if $a, b, c$ are coplanar. If $a, b, c$ satisfy (18), then for each of the angles we have only two possibilities:

$$
\widehat{a b}=\alpha \text { or } \widehat{b c}=\beta \text { or } 2 \pi-\beta, \quad \widehat{c a}=\gamma \text { or } 2 \pi-\gamma
$$

Because of (11), $2 \pi-\alpha, 2 \pi-\beta, 2 \pi-\gamma \geq \pi$ therefore, because of (23), the second possibility can take place at most for one of the angles. Let now $a^{\prime}, b^{\prime}, c^{\prime} \in S^{(2)}$ be another triple of vectors satisfying (18). Put $\theta_{1}=\widehat{a b}, \theta_{2}=\widehat{b c}, \theta_{3}=\widehat{c a}$ and $\theta_{j}^{\prime}$ the corresponding angles for $a^{\prime}, b^{\prime}, c^{\prime}$. If (21) does not hold, then for the reasoning above there is at most an index-let us call it $i$-such that $\theta_{i}^{\prime}=2 \pi-\theta_{i}$. Denoting $j, k$ the remaining indices, one has, from (23) and (22),

$$
2 \pi \geq \theta_{i}^{\prime}+\theta_{j}^{\prime}+\theta_{k}^{\prime}=2 \pi-\theta_{i}+\theta_{j}+\theta_{k} \geq 2 \pi-\theta_{i}+\theta_{i}=2 \pi .
$$

Thus $a^{\prime}, b^{\prime}, c^{\prime}$ must be coplanar. But in this case from $\left|\theta_{i}^{\prime}-\theta_{j}^{\prime}\right| \leq \theta_{k}^{\prime}$ we deduce $2 \pi \leq$ $\left(\theta_{i}+\theta_{j}+\theta_{k}\right) \leq 2 \pi$, hence also $a, b, c$ must be coplanar. If $a^{\prime}, b^{\prime}, c^{\prime}$ are not coplanar the first inequality in (25) is strict, and this contradicts the last inequality. Hence for no index $i$ we can have $\theta_{i} \neq \theta_{i}^{\prime}$ and this proves $(21)$.

(13) F. Enriquez and U. Amaldi: Elementi di geometira (Bologna, 1921). 
Complex Hilbert-space models for $P, Q, R$. - Assume that there exists a complexHilbert-spce model for $P, Q, R$. Then, there are a Hilbert space $\mathcal{H} \cong \mathbf{C}^{2}$; three orthonormal basis $\left(\varphi_{\alpha}\right)\left(\psi_{\beta}\right),\left(\chi_{\beta}\right)$ satisfying $(5)$; and real numbers $\varrho_{\alpha \beta}(A, B), \varrho_{\beta \gamma}(B, C)$, $\varrho_{\gamma \alpha}(C, A)$ such that

$$
\left.\left\langle\varphi_{\alpha}, \psi_{\beta}\right\rangle=\exp \left[i \varrho_{\alpha \beta}(A, B)\right] \sqrt{P\left(A=a_{\alpha} \mid B=b_{\beta}\right.}\right), \ldots .
$$

Therefore using the orthogonality relation

$$
\left\langle\varphi_{\alpha}, \chi_{\gamma}\right\rangle=\sum_{\beta}\left\langle\varphi_{\alpha}, \psi_{\beta}\right\rangle\left\langle\psi_{\beta}, \chi_{\gamma}\right\rangle
$$

one immediately verifies the equality

$$
\begin{gathered}
\exp \left[-i \varrho_{11}(C, A)\right] \cos \gamma / 2=\exp \left[i\left[\varrho_{11}(A, B)+\varrho_{11}(B, C)\right]\right] \cos \alpha / 2 \cos \beta / 2+ \\
\cdot \exp \left[i\left[\varrho_{12}(A, B)+\varrho_{21}(B, C)\right]\right] \sin \alpha / 2 \sin \beta / 2 .
\end{gathered}
$$

Lemma 5. Let $\lambda_{1}, \lambda_{2}, \lambda_{3}$ be positive real numbers. A necessary and sufficient condition for the existence of real numbers $x, y, z$, which solve the equation

$$
\lambda_{1} \exp [i x]+\lambda_{2} \exp [i y]=\lambda_{3} \exp [i z],
$$

is that

$$
-1 \leq \frac{\lambda_{3}^{2}-\left(\lambda_{1}^{2}+\lambda_{2}^{2}\right)}{2 \lambda_{1} \lambda_{2}} \leq 1
$$

or equivalently, that

$$
-1 \leq \frac{\lambda_{1}^{2}+\lambda_{3}^{2}-\lambda_{2}^{2}}{2 \lambda_{1} \lambda_{3}} \leq 1
$$

Proof.- If a solution of (28) exists, then

$$
\cos (x-y)=\frac{\lambda_{3}^{2}-\left(\lambda_{1}^{2}+\lambda_{2}^{2}\right)}{2 \lambda_{1} \lambda_{2}}
$$

and (29) is satisfied. Conversely, if (29) holds one can choose an arbitrary couple $x, y$ such that (31) is satisfied, and with this choice both sides of the equality (28) have the same modulus, thus a solution of (28) exists. Finally, the solvability of (28) is equivalent to the solvability of

$$
\lambda_{1} \exp [i x]-\lambda_{3} \exp [i z]=-\lambda_{2} \exp [i y]
$$


and, writing condition (29) — with the appropriate coefficients - for eq. (32) one finds (30).

Remark. - One easily verifies that an equivalent condition for the existence of solutions of eq. (28) is that the inequality $\lambda_{1} \leq \lambda_{2}+\lambda_{3}$ and those obtained from this by circular permutation hold. This from of the condition, as we will show elsewhere, has a more general character than (29) or (30), but in the present paper only this last type of inequalities will be used.

Corollary 6 . The following equivalent inequalities are necessary conditions for the existence of a complex-Hilbert-space model for the transition matrices $P, Q, R$ :

$$
\begin{gathered}
\cos ^{2} \alpha+\cos ^{2} \beta+\cos ^{2} \gamma-1 \leq 2 \cos \alpha \cos \beta \cos \gamma \\
-1 \leq \frac{\cos ^{2} \alpha / 2+\cos ^{2} \beta / 2+\cos ^{2} \beta / 2-1}{2 \cos \alpha / 2 \cos \beta / 2 \cos \gamma / 2} \leq 1 \\
{[\cos \alpha / 2 \cos \beta / 2-\sin \alpha / 2 \sin \beta / 2]^{2} \leq \cos ^{2} \gamma / 2 \leq} \\
\leq[\cos \alpha / 2 \cos \beta / 2+\sin \alpha / 2 \sin \beta / 2]^{2} .
\end{gathered}
$$

Proof .- If a Hilbert space model for $P, Q, R$ exists, then (27) holds and therefore eq. (28) with th coefficients

$$
\lambda_{3}=\cos \gamma / 2, \quad \lambda_{1}=\cos \alpha / 2 \cos \beta / 2, \quad \lambda_{3}=\sin \alpha / 2 \sin \beta / 2
$$

must be solvable. With this choice of the coefficients the solvability condition (29) becomes

$$
-\sin \alpha \sin \beta \leq \cos \gamma-\cos \alpha \cos \beta \leq \sin \alpha \sin \beta
$$

and this is an equivalent formulation of (35).

Because of (11) the sines are positive, hence (36) is equivalent to

$$
|\cos \gamma-\cos \alpha \cos \beta|^{2} \leq \sin ^{2} \alpha \sin ^{2} \beta
$$

which is easily seen to be equivalent to (33). If, instead of the solvability condition (29), the equivalent condition (30) is used with the same coefficients $\lambda_{1}, \lambda_{2}, \lambda_{3}$, the results is $(34)$.

Theorem 7. The following assertions are equivalent:

i) the transition matrices $P, Q, R$ admit a complex-Hilbert-space model

ii) the transition matrices $P, Q, R$ admit a spin model;

$$
\text { iii) } \cos ^{2} \alpha+\cos ^{2} \beta+\cos ^{2} \gamma-1 \leq 2 \cos \alpha \cos \beta \cos \gamma \text {; }
$$




$$
\begin{gathered}
\text { iv) }-1 \leq \frac{\cos ^{2} \alpha / 2+\cos ^{2} \beta / 2+\cos ^{2} \gamma / 2-1}{2 \cos \alpha / 2 \cos \beta / 2 \cos \gamma / 2} \leq 1 ; \\
\text { v) }-1 \leq \frac{p+q+r-1}{2 \sqrt{p q r}} \leq 1 ; \\
\text { vi) }[\sqrt{p q}-\sqrt{(1-p)(1-q)}]^{2} \leq r \leq[\sqrt{p q}+\sqrt{(1-p)(1-q)}]^{2} .
\end{gathered}
$$

Proof.- The equivalence iii) $\Leftrightarrow$ iv) has been shown in Corollary 6 and v) is just iv) in different notations. That iii) $\Leftrightarrow$ ii) is the content of Proposition 3. Clearly ii) $\Rightarrow$ (i). But, because of Corollary 6, i) $\Rightarrow$ iii) and hence ii). The equivalence v) $\Leftrightarrow$ vi) has been established in Corollary 6.

Corollary 8. i) Every complex-Hilbert-space model for $P, Q, R$ is a spin model. ii) If a spin model for $P, Q, R$ is defined by three noncoplanar vectors $a, b, c \in \mathbf{R}^{3}$, then all spin models are unitarily equivalent (in the sense that there exists a single unitary operator which maps the three orthonormal basis of one model into chose of the other one).

Proof.- If $\left(\varphi_{\alpha}\right),\left(\psi_{\beta}\right),\left(\chi_{\gamma}\right)$ are any three orthonormal basis of $\mathcal{H}$ which realize a complex Hilbert space model of $P, Q, R$, then by Theorem 7 there are in $\mathcal{H}$ three orthonormal basis of spin type $\left(\psi_{\alpha}(a)\right),\left(\psi_{\beta}(b)\right),\left(\psi_{\gamma}(c)\right)$ with the same property. If $U_{1}, U_{2}, U_{3}$ are three unitaries such that

$$
U_{1} \psi_{\alpha}(a)=\varphi_{\alpha}, U_{2} \psi_{\beta}(b)=\psi_{\beta}, \quad U_{3} \psi_{\gamma}(c)=\chi_{\gamma}
$$

they define three rotations $R_{1}, R_{2}, R_{3}$ such that

$$
\psi_{\alpha}\left(R_{1} a\right)=\varphi_{\alpha} ; \quad \psi_{\beta}\left(R_{2} b\right)=\psi_{\beta} ; \quad \psi_{\gamma}\left(R_{3} c\right)=\chi_{\gamma}
$$

and this proves i). If $a, b, c$ are not coplanar then, denoting $a^{\prime}=R_{1} a, b^{\prime}=R_{2} b ; c^{\prime}=R_{3} c$, Proposition 4 implies that $\widehat{a b}=\widehat{a^{\prime} b^{\prime}}, \widehat{b c}=\widehat{b^{\prime} c^{\prime}}, \widehat{c a}=\widehat{c^{\prime} a^{\prime}}$ hence there is a rotation $R$ in $\mathbf{R}^{3}$ such that $R a=a^{\prime}, R b=b^{\prime}, R c=c^{\prime}$. Therefore in (39) the three unitaries can be taken equal.

Remark. It is easy to verify that because of (11) condition (37) is equivalent to the following couple of inequalities:

$$
|\alpha-\beta| \leq \gamma \leq \alpha+\beta, \quad \alpha+\beta+\gamma \leq 2 \pi,
$$

which are necessary and sufficient conditions on $\alpha, \beta, \gamma$ to be adjacent angles of a trihedron in $\mathbf{R}^{3}$. This provides a geometrical interpretation for condition (37).

Real-Hilbert-space models for $P, Q, R$. 
Theorem 9. The transition matrices $P, Q, R$ admit a real-Hilbert-space model, if and only if they admit a spin model defined by a coplanar triple of vectors $a, b, c \in S^{(2)}$.

Proof. Sufficiency. If the transition matrices $P, Q, R$ admit a spin model defined by a coplanar triple of vectors $a, b, c \in S^{(2)}$, then by Proposition 4 all triples are coplanar and in appropriate co-ordinates in $\mathbf{R}^{3}$ the vectors $a, b, c$ will have the form

$$
a=\left(a_{1}, 0, a_{3}\right), \quad b=\left(b_{1}, 0, b_{3}\right), \quad c=\left(c_{1}, 0, c_{3}\right) .
$$

But in a basis of $\mathcal{H}$ in which the Pauli matrices have the usual from (15), the eigenvectors of $\sigma \cdot x\left(x=\left(x_{1}, x_{2}, x_{3}\right) \in \mathbf{R}^{3}\right)$ have components (defined up to a common phase)

$$
\psi_{1}(x)=\left(\begin{array}{c}
\frac{\sqrt{\left(1+x_{3}\right) / 2}}{x_{1}+i x_{2}} \\
\sqrt{2\left(1+x_{3}\right)}
\end{array}\right), \quad \psi_{2}(x)=\left(\begin{array}{c}
\sqrt{\left(1-x_{3}\right) / 2} \\
-\frac{x_{1}+i x_{2}}{\sqrt{2\left(1-x_{3}\right)}}
\end{array}\right),
$$

hence for each vector of the form (43) the phase can be chosen so that it has real components. With this choice of the phase the vectors of the two basis $\left(\psi_{\beta}(b)\right),\left(\psi_{\gamma}(c)\right)$ belong to the real Hilbert space generated by the basis $\left(\psi_{\alpha}(a)\right)$, hence this space provides a model for $P, Q, R$.

Necessity. Assume that the transition matrices $P, Q, R$ admit a real-Hilbert-space model. Then they admit a complex-Hilbert-space model and, by Corollary 8 , a spin model. Let $\left(\psi_{\alpha}(a)\right),\left(\psi_{\beta}(b)\right),\left(\psi_{\gamma}(c)\right)$ be a triple of orthonormal basis which define this model $(a, b, c \in$ $\left.S^{(2)}\right)$. Then, because of our assumption, (27) must hold with the phasis which are integer multiples of $\pi$. Using conditions (29) and (30) with the notations

$$
\lambda_{3}=\cos \gamma / 2, \quad \lambda_{1}=\cos \alpha / 2 \cos \beta / 2, \quad \lambda_{2}=\sin \alpha / 2 \sin \beta / 2,
$$

one easily verifies that at least one of the following equalities must be satisfied:

$$
\text { 1) } \lambda_{1}+\lambda_{2}=\lambda_{3}, \text { 2) }-\lambda_{1}+\lambda_{2}=\lambda_{3}, \text { 3) } \lambda_{1}-\lambda_{2}=\lambda_{3}
$$

and, because of (44), the equalities (45) are, respectively, equivalent to

$$
\text { 1) } 2 \pi-\alpha+\beta+\gamma=2 \pi, \quad \alpha+\beta+\gamma=2 \pi, \quad \alpha+\beta+2 \pi-\gamma=2 \pi \text {, }
$$

Because of (24) any of the equalities (46) will yield

$$
\widehat{a b}+\widehat{b c}+\widehat{c a}=2 \pi .
$$

Hence $a, b, c$ are coplanar.

Proof of Theorem 9. In the notations (44), the transition matrices $P, Q, R$ admit a real-Hilbert-space model if and only the equation

$$
\lambda_{1} \exp [i x]+\lambda_{2} \exp [i y]=\lambda_{3} \exp [i z]
$$


has a solution such that $x, y, z$ are integer multiples of $\pi$. From the proof of Lemma 5 it follows that such a solution exists, if and only if, for some integer $k$

$$
\cos (k \pi)=\frac{\lambda_{1}^{2}+\lambda_{3}^{2}-\lambda_{2}^{2}}{2 \lambda_{1} \lambda_{3}}
$$

and this is equivalent to (42).

Concluusions.- Let us denote (Kolm), (C-Hilbert), (R-Hilbert) the family of triples $P, Q$, $R$ of bi-stochastic matrices of the form (10) which admit respectively a Kolmogorovian, a complex-Hilbert-space, a real-Hilbert-space model. Clearly $(\mathbf{R}$-Hilbert $) \subseteq(\mathbf{C}-$ Hilbert $)$, and Theorem 7 and 9 show that the inclusion is strict. The elementary inequalities

$$
[\sqrt{p q}-\sqrt{(1-p)(1-q)}]^{2} \leq|p+q-1| \leq 1-|p-q| \leq[\sqrt{p q}+\sqrt{(1-p)(1-q)}]^{2}
$$

together with Theorems 2 and 7 , show that also the inclusion (Kolm) $\subseteq(\mathbf{C}-$ Hilbert) takes place. Since, in the first inequality the equality sign holds if and only if $p+q=1$ and in the third one if and only if $p=q$, also in this case the inclusion is strict. In particular, if the three matrices $P, Q, R$ correspond to a spin model defined by three noncoplanar vectors $a, b, c \in S^{(2)}$ and are such that either

$$
\begin{gathered}
{[\sqrt{p q}-\sqrt{(1-p)(1-q)}]^{2}<r<|p+q-1| \text { or }} \\
1-|p-q|<r<[\sqrt{p q}+\sqrt{(1-p)(1-q)}]^{2}
\end{gathered}
$$

then they will not admit neither a Kolmogorovian nor a real-Hilbert-space model but, by construction, they admit a complex-Hilbert-space model.

While the inclusion $(\mathbf{R}-$ Hilbert $) \subseteq(\mathbf{C}-$ Hilbert $)$ is of general character, the inclusion $($ Kolm $) \subseteq(\mathbf{C}-$ Hilbert $)$ depends strongly on the fact that we are considering 2 -valued observables.

For three (or $\geq 3$ ) valued observables this inclusion fails already at the level of pairs of observables, i.e. in the case of a single bistochastic matrix. For example, it can be shown (cf. $^{(3)}$ ) that if $0<\delta<1 / 2^{6}$ the bistochastic matrix

$$
\left[\begin{array}{ccc}
\frac{1}{2}\left(\frac{1}{2}+\delta\right) & \frac{1}{2^{4}} & \frac{11}{2^{4}}-\frac{1}{2} \delta \\
\frac{1}{2}\left(\frac{1}{2}+\delta\right) & \frac{1}{2}-\frac{1}{2^{4}}+\delta & 1-2^{\delta}-\left(\frac{11}{2^{4}}-\frac{1}{2} \delta\right) \\
\frac{1}{2}-\delta & \frac{1}{2}-\delta & 2 \delta
\end{array}\right]
$$

(which, of course, admits infinitely many Kolmogorovian models)

does not admit a complex-Hilbert-space model.

Remark. Our Theorem 2 is equivalent to a result established by D. Gutkowski and G. Masotto $^{(14)}$ (necessary condition) and by G. Corleo, G. Gutkowski, G. Masotto, M.V.

(14) D. Gutkowski and G. Masotto: Nuovo Cimento D, 22, 121 (1974). 
Valdes ${ }^{(15)}$ (sufficient condition). Their result is expressed in terms of joint rather than conditional probabilities, and our inequality (12) is obtained by their inequality (1) (in ${ }^{(15)}$ ) just dividing each term by $\frac{1}{2}$. The factor $\frac{1}{2}$ is canonical since, due to the symmetry condition (2), any Kolmogorovian model for the given set of transition probabilities must satisfy

$$
\mu\left(A=a_{\alpha}\right)=\mu\left(B=b_{\beta}\right)=\mu\left(C=c_{\gamma}\right)=\frac{1}{2}, \quad \alpha, \beta, \gamma=1,2 .
$$

The equivalence of our Theorem 2 with the mentioned results of Gutkowski et al. was kindly pointed out to us by prof. R. Ascoli, to whom we want to express our gratidue for this as well as for other interesting remarks. Let us also remark that a necessary condition on the 2-dimensional joint probabilities of any finite number of 2-valued observables, for the existence of a Kolmogorovian model, has been established by A. Garuccio and F. Selleri ${ }^{(16)}$.

(15) G. Corleo, D. Gutkowski, G. Masotto and M.V. Valdes: Nuovo Cimento B, 25, 413 (1975).

(16) A. Garuccio and F. Selleri: Found. Phys., 10, 209 (1980). 